\title{
On Symmetric, Orthogonal, and Skew-Symmetric Matrices
}

\author{
By P. L. Hsu
}

(Received 9th July 1948. Read 5th November 1948.)

1. Introduction and Notation. In this paper all the scalars are real and all matrices are, if not stated to be otherwise, $p$-rowed square matrices. The diagonal and superdiagonal elements of a symmetric matrix, and the superdiagonal elements of a skew-symmetric matrix, will be called the distinct elements of the respective matrices. $\Sigma$ will denote both the set of all symmetric matrices and the $\frac{1}{2} p(p+1)$-dimensional space whose coordinates are the distinct elements arranged in some specific order. $K$ will denote both the set of all skew-symmetric matrices and the $\frac{1}{2} p(p-1)$-dimensional space whose coordinates are the distinct elements arranged in some specific order. Any sub-set of $\Sigma(K)$ will mean both the sub-set of symmetric (skew-symmetric). matrices and the set of points of $\Sigma(K)$. Any point function defined in $\Sigma(K)$ will be written as a function of a symmetric (skew-symmetric) matrix. $D_{a}$ will denote the diagonal matrix whose diagonal elements are $a_{1}, a_{2}, \ldots, a_{p}$. The characteristic roots of a symmetric matrix will be called its roots.

The orthogonal matrices $\Gamma$ with $|\Gamma+I| \neq 0$ and the skewsymmetric matrices $X$ are in $(1,1)$-correspondence, on account of the following pair of equivalent equations :

$$
\begin{aligned}
& \Gamma=2(I+X)^{-1}-I, \\
& X=2(I+\Gamma)^{-1}-I .
\end{aligned}
$$

That the skew-symmetry of $X$ implies the orthogonality of $\Gamma$, and vice versa, ${ }^{1}$ is the direct result of the following computation:

$$
\begin{aligned}
& {\left[2(I+X)^{-1}-I\right]\left[2(I+X)^{-1}-I\right]^{\prime}} \\
& =(I+X)^{-1}(I-X)(I+X)(I-X)^{-1} \\
& =(I+X)^{-1}(I+X)(I-X)(I-X)^{-1}=I \text {, } \\
& {\left[2(I+\Gamma)^{-1}-I\right]+\left[2(I+\Gamma)^{-1}-I\right]^{\prime}} \\
& =2(I+\Gamma)^{-1}+2(I+\Gamma)^{-1} \Gamma-2 I=2(I+\Gamma)^{-1}(I+\Gamma)-2 I=0 \text {. }
\end{aligned}
$$

If $S \in \Sigma$, and if its roots are $\theta_{1}, \ldots, \theta_{p}$, it is well known that

$$
S=\Delta D_{\theta} \Delta^{\prime}
$$

where $\Delta$ is some orthogonal matrix.

1 This result, together with a lengthy derivation, is given in Kowalewski, Einführung in die Determinantentheorie (Leipzig, 1909), pp. 171-175. 
Lемма 1. If $A$ is any $p \times p$ matrix, there is a matrix $D_{\epsilon}$, with $\epsilon_{i}=+1$ or $-1(i=1, \ldots, p)$, such that $\left|A+D_{\mathrm{\epsilon}}\right| \neq 0$.

Proof. The lemma is evidently true for $p=1$. Assume it is true for $p-1$. Write

$$
A=\left(\begin{array}{ll}
A_{1} & b^{\prime} \\
c & d
\end{array}\right)
$$

where $A_{1}$ is $(p-1) \times(p-1)$ and $b, c$ are rows. By assumption there is a $D_{\eta}$, with $\eta_{i}=+1$ or $-1(i=1, \ldots, p-1)$, such that $\left|A_{1}+D_{\eta}\right| \neq 0$. Since

$$
\left|\begin{array}{rr}
A_{1}+D_{\eta} & b^{\prime} \\
c & d+1
\end{array}\right|-\left|\begin{array}{rr}
A_{1}+D_{\eta} & b^{\prime} \\
c & d-1
\end{array}\right|=2\left|A_{1}+D_{\eta}\right| \neq 0,
$$

the two determinants on the left side of (3) cannot both vanish. Hence the lemma is proved.

Lemma 2. If $S \in \Sigma$, there is an orthogonal $\Gamma$ with $|\Gamma+I| \neq 0$ such that $S=\Gamma D_{\theta} \Gamma^{\prime}$.

Proof. For the $\Delta$ in (2) there is, by Lemma 1, a $D_{e}$ with $\left|\Delta+D_{\epsilon}\right| \neq 0$. Hence $\left|\Delta D_{\epsilon}+I\right| \neq 0$. Moreover, $\Delta D_{\varepsilon}$ also satisfies (2). Hence the matrix $\Gamma=\Delta D_{\varepsilon}$ answers all the requirements.

Combining Lemma 2 with (1) we obtain

Theorem 1. If $S \in \Sigma$, there is an $X \in K$ such that

$$
S=\left[2(I+X)^{-1}-I\right] D_{\theta}\left[2(I+X)^{-1}-I\right]^{\prime} \text {. }
$$

In $\S 2$ we investigate the uniqueness of the expression (4) for a given $S$. (4) expresses each distinct element of $S$ as a function of $\frac{1}{2} p(p+1)$ arguments, viz., the distinct elements of $X$ and the $\theta$ 's. In $\S 3$ we evaluate the functional determinant of these functions or, to use another term, the Jacobian of the transformation (4). In $\S 4$ we apply the results to prove a formula for the integral of some function of $S$.

2. The symmetric matrices $S$ which have multiple roots satisfy the equation $f(S)=0$, where $f(S)$ is the discriminant of the characteristic equation of $S$. Let $F$ be the surface $f(S)=0$. If $S \in \Sigma-F$, 
we shall so name the roots $\theta_{1}, \ldots, \theta_{p}$ that $\theta_{1}>\theta_{2}>\ldots>\theta_{p}$. Thus each $\theta_{i}$ is a well-defined function of $S$. The equation

$$
S=\Gamma^{\prime} D_{\theta} \Gamma^{\prime},
$$

where $\Gamma=\left(\gamma_{i j}\right)$ is orthogonal, then uniquely determines every colum $n$ of $\Gamma$ except for a sign. In particular,

$$
\left|\gamma_{1 i}\right|=g_{i}(S) \quad(i=2, \ldots, p)
$$

are well-defined functions of $S$. Let $F_{i}$ be the surface $g_{i}(S)=0$. If $S \in \Sigma-F-F_{2}-\ldots-F_{p}$, we take $\gamma_{1 i}=-g_{i}(S)(i=2, \ldots, p)$. Thus all the columns except the first one of $\Gamma$ are uniquely determined. since the signs of the top elements of these columns are determined. Hence we have

Lemma 3. If $S \in \Sigma-F-F_{2}-\ldots-F_{p}$, equation (5) has exactly two solutions for $\Gamma: \Gamma_{1}$ and $\Gamma_{2}$, whose elements $(1,2),(1,3), \ldots,(1, p)$, are all negative. $\Gamma_{1}$ and $\Gamma_{2}$ differ only in the respect that the first column of one is the negative of the first column of the other.

By virtue of Lemma 3 all the elements $\gamma_{i j}(i, j=2, \ldots, p)$ of $\Gamma$ are well-defined functions of $S$. Writing $\Gamma^{(1)}=\left(\gamma_{i j}\right)_{(i, j=2 \ldots, p)}$ we have $\left|\Gamma^{(1)}+I\right|=h(S)$, a well-defined function of $S$. Let $F^{\prime}$ be the surface $h(S)=0$. Let $E=\Sigma-F^{\prime}-F_{2}-\ldots-F_{p}-F^{\prime}$.

Lemma 4. If $S \in E$, one of the determinants $\left|\Gamma_{1}+I\right|$ and $\left|\Gamma_{2}+I\right|$ in Lemma 3 is zero while the other is not zero.

Proof. By Lemma 3 and the definition of $E$ we have

$$
\left|\Gamma_{1}+I\right|+\left|\Gamma_{2}+I\right|=2\left|\Gamma^{(1)}+I\right| \neq 0 \text {. }
$$

Hence one of the determinants is not zero. Suppose $\left|\Gamma_{1}+I\right| \neq 0$. Then, by (1), $\Gamma_{1}=2(I+X)^{-1}-I$, where $X$ is skew-symmetric. Letting $H$ be the diagonal matrix $[-1,1, \ldots, 1]$ we have $\Gamma_{2}=\Gamma_{1} H$. Hence

$$
\begin{aligned}
\left|\Gamma_{2}+I\right|=\left|\Gamma_{1} H+I\right| & =\left|2(I+X)^{-1} H+I-H\right| \\
& =|X+I|^{-1}|2 H+(I+X)(I-H)| .
\end{aligned}
$$

It is easily seen that the first row of the matrix $2 H+(I+X)(I-H)$. consists of zeros. Hence $\left|\Gamma_{2}+I\right|=0$. This completes the proof.

If we take $\Gamma$ to be $\Gamma_{1}$ or $\Gamma_{2}$ according as $\left|\Gamma_{1}+I\right| \neq 0$ or $\left|\Gamma_{2}+I\right| \neq 0$ we obtain

Lemma 5. If $S \in E$, there is a unique $\Gamma$ which satisfies (5) and the following conditions : 


$$
\begin{array}{ll}
\text { (i) } & \gamma_{1 i}<0 \quad(i=2, \ldots, p) \\
\text { (ii) } 1 \Gamma+I: \neq 0 .
\end{array}
$$

By combining Lemmn 5 with (1) and noticing that in (1) the nondiagonal elements of $\Gamma$ and $(I+X)^{-1}$ must have the same sign, we obtain

THEOREM 2. If $S \in E$, the equation (4) has a unique solution in $X$ such that the elements $(1,2),(1,3), \ldots,(1, p)$ of $(I+X)^{-1}$ are all negative.

Let $M$ be the sub-set of $K$ defined by the condition that the elements $(1,2),(1,3), \ldots,(1, p)$ of $(I+X)^{-1}$ are all negative, let $\Theta$ be the sub-set of the space of $\left(\theta_{1}, \ldots, \theta_{p}\right)$ defined by the condition $\theta_{1}>\theta_{2}>\ldots>\theta_{p}$, and let $E^{*}=M \times \Theta$, a sub-set of the ${ }_{2}^{1} p(p+1)$-dimensional space. Theorem 2 asserts that the equation (4) effects a (1, 1)-mapping of $E^{*}$.on $E$. Notice also that by the definition of $E$, it differs from $\Sigma$ by a set of measure zero.

3. In order to facilitate the computation of the functional determinant ${ }^{1}$ for (4) we shall adopt the following notation. If $x=\left(x_{1}, \ldots, x_{m}\right)$ and $y=\left(y_{1}, \ldots, y_{m}\right)$ and if

$$
x_{i}=\sum_{j=1}^{m} a_{i j} y_{j} \quad(i=1, \ldots, m)
$$

then we define the symbol $D(x ; y)$ to be the discriminant $\left|a_{i j}\right|$.

Lemma 6.

$$
\begin{aligned}
\text { (i) } \quad D(x ; y) & =\frac{1}{D(y ; x)} \\
\text { (ii) } \quad D(x ; y) & =D(x ; z) D(z ; y) .
\end{aligned}
$$

Proof. (i) is an immediate consequence of definition; (ii) is a special case of the multiplicative law of functional determinants.

The extension of (ii) to more than two factors is obvious.

In the equation

$$
X=A Y A^{\prime},
$$

$X$ is symmetric or skew-symmetric according as $Y$ is symmetric or skew-symmetric. In either case (6) expresses each distinct element of $X$ as a linear function of the distinct elements of $Y$, with coefficients depending on $A$. The following lemma gives the discriminant $D(X ; Y)$ of this set of linear functions.

1 Functional determinants are considered as deternined up to a sign. In all the computations in this section signs of functional determinants are neglected. 
On Symmetric, Orthogonal, and Skew-Symuetric Matrices 41

Lemia 7. For (6) we have

$$
D(X ; Y)=\begin{array}{ll}
|A|^{p+1} & \text { if } Y \text { is symmetric } \\
|A|^{p-1} & \text { if } Y \text { is skew-symmetric. }
\end{array}
$$

Proof. It is sufficient to prove (7) for a non-singular $A$. Now every non-singular matrix is a product of a finite number of matrices of either of the following two types, (a) a diagonal matrix whose diagonal elements are 1 with the exception of one, which is, say, $a$; (b) a matrix whose diagonal elements are 1 and whose non-diagonal elements are 0 with the exception of one. Further, if we denote $D(X ; Y)$ for $(6)$ by $P(A)$, a moment of reflection will show that $P(A B)=P(A) P(B)$. Hence it is sufficient to prove (7) for $A$ belong-

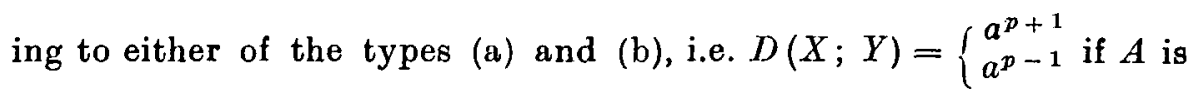
of type (a) and $D(X ; Y)=1$ for both cases if $A$ is of type (b). The proof of these assertions is easy and is left to the reader.

If $x_{1}, \ldots, x_{m}$ are functions of $y_{1}, \ldots, y_{m}$, then by definition the functions d determinant is equal to $D(d x ; d y)$, where $d x$ denotes the system $d x_{1}, \ldots, d x_{n}$. For brevity we write $d A$ for the matrix whose elements are the differentials of the elements of $A$.

In order to differentiate (1) we use the formula

$$
d A^{-1}=-A^{-1}(d A) A^{-1} .
$$

Then

$$
d \Gamma=-2(I+X)^{-1} d X(I+X)^{-1}=-\frac{1}{2}(I+\Gamma) d X(I+\Gamma) .
$$

Let $J$ be the functional determinant for (4), i.e. for

where $\Gamma$ is given by (1). Differentiating (9) and using (8) we have

Also,

$$
\begin{array}{r}
d S=\Gamma D_{\theta} d \Gamma^{\prime}+(d \Gamma) D_{\theta} \Gamma^{\prime}+\Gamma D_{\theta} \Gamma^{\prime}=\frac{1}{2} \Gamma D_{\theta}\left(I+\Gamma^{\prime}\right) d X\left(I+\Gamma^{\prime}\right) \\
-\frac{1}{2}(I+\Gamma) d X(I+\Gamma) D_{\theta} \Gamma^{\prime}+\Gamma D_{d \theta} \Gamma^{\prime} .
\end{array}
$$

From (10) we get

(12) $\Gamma^{\prime} d S \Gamma=\frac{1}{2} D_{\theta}\left(I+\Gamma^{\prime}\right) d X(I+\Gamma)-\frac{1}{2}\left(I+\Gamma^{\prime}\right) d X(I+\Gamma) D_{\theta}+D_{d \theta}$.

If we write $\xi_{i}$ for $d \theta_{i}$, and define $U$ and $Y$ by

$$
\begin{gathered}
U=\Gamma^{\prime} d S \Gamma \\
Y=\left(I+\Gamma^{\prime}\right) d X(I+\Gamma),
\end{gathered}
$$


then (12) gives

i.e.,

$$
U=\frac{1}{2} D_{\theta} Y-\frac{1}{2} Y D_{\theta}+D_{\xi},
$$

$$
\begin{array}{ll}
u_{i i} & =\xi_{i} \quad(i=1, \ldots, p), \\
u_{i j} & =\frac{1}{2}\left(\theta_{i}-\theta_{j}\right) y_{i j} \quad(i<j) .
\end{array}
$$

(13), (14) and (15) give respectively

$$
\begin{aligned}
& D(d S ; U)=\frac{1}{D(U ; d S)}=1, \text { by Lemmas } 6 \text { and } 7 \\
& D(Y ; d X)=2^{p(p-1)}|I+X|^{-(p-1)}, \text { by Lemma } 6 \text { and }(1) ; \\
& D(U ; Y, \xi)=2^{-\frac{1}{2} p(p-1)} \prod_{i<j}\left(\theta_{i}-\theta_{j}\right)
\end{aligned}
$$

Hence, by (11) and Lemima 7,

$$
\begin{aligned}
J & =D(d S ; U) D(U ; Y, \xi) D(Y, \xi ; d X, \xi) \\
& =D(d S ; U) D(U ; Y, \xi) D(Y ; d X),
\end{aligned}
$$

and so, finally,

$$
J=2^{\mathfrak{1} p(p-1)}|I+X|^{-(p-1)} \prod_{i<j}\left(\theta_{i}-\theta_{j}\right) .
$$

4. Let us first evaluate the following integrals:

$$
\begin{aligned}
& A_{p}=\int_{K}|I+X|^{-(p-1)} d m \\
& B_{p}=\int_{M}|I+X|^{-(p-1)} d m
\end{aligned}
$$

where $X$ is a $p$-rowed skew-symmetric matrix, $d m$ denotes the volumeelement, $K$ is the $\frac{1}{2} p(p-1)$-dimensional space, and $M$ is defined in the last paragraph of $\S 2$.

We write

so that

$$
X=\left(\begin{array}{cc}
0 & x \\
-x^{\prime} & Y
\end{array}\right), \quad x=\left(x_{1}, \ldots, x_{p-1}\right)
$$

$$
|I+X|=\left|\begin{array}{cc}
1 & X \\
-x^{\prime} & I+Y
\end{array}\right|=|I+Y|\left(1+x(I+Y)^{-1} x^{\prime}\right) .
$$

Also, the elements $(1,2),(1,3), \ldots,(1, p)$ of $(I+X)^{-1}$ are the elements . of the row $-\left(1+x(I+Y)^{-1} x^{\prime}\right)^{-1} x(I+Y)^{-1}$. Hence $M$ is the set. of points such that every element of the row $x(I+Y)^{-1}$ is positive. Making the transformation

$$
x^{\prime}=(I+Y) u^{\prime}, \quad u=\left(u_{1}, \ldots, u_{p-1}\right)
$$

whose Jacobian is $|I+Y|$, we obtain 
On Symmetric, Orthogonal, and Skew-Symmetric Matrices 43 $x(I+Y)^{-1} x^{\prime}=u(I+Y) u^{\prime}=u u^{\prime}=u_{1}^{2}+\ldots+u_{p-1^{2}}$.

Hence

$$
\begin{aligned}
& A_{p}=\int_{K 1}|I+Y|-(p-2) d m \int_{-\infty}^{\infty} \ldots \int_{-\infty}^{\infty}\left(1+u_{1}^{2}+\ldots\right. \\
& \left.+u_{p-1}^{2}\right)^{-(p-1)} d u_{1} \ldots d u_{p-1} \text {, } \\
& \text { (20) } \quad B_{p}=\int_{K_{1}}|I+Y|^{-(p-2)} d m \int_{0}^{\infty} \ldots \int_{0}^{\infty}\left(1+u_{1}^{2}+\ldots\right. \\
& \left.+u_{p-1}^{2}\right)^{-(p-1)} d u_{1} \ldots d u_{p-1} \text {, }
\end{aligned}
$$

where $K_{1}$ is the $\frac{1}{2}(p-1)(p-2)$-dimensional space. It follows from (19) and (20) that

$$
\text { (22) } \begin{aligned}
A_{p} & =A_{p-1} \int_{-\infty}^{\infty} \cdots \int_{-\infty}^{\infty}\left(1+u_{1}^{2}+\ldots+u_{p-1}^{2}\right)^{-(p-1)} d u_{1} \ldots d u_{p-1} \\
& =\frac{\pi^{p / 2}}{2^{p-2} \Gamma(p / 2)} A_{p-1},
\end{aligned}
$$

and the easy computation $A_{2}=\pi$ leads to the result

$$
A_{p}=\pi \prod_{r=3}^{p} \frac{\pi^{r / 2}}{2^{r-2} \Gamma(r / 2)}=\frac{\pi^{p(p+1) / 4}}{2^{(p-1)(p-\overline{2) / 2}} \prod_{r=1}^{p} \Gamma(r / 2)},
$$

whence also

$$
B_{p}=\frac{\pi^{p(p+1) / 4}}{2^{p(p-1) / 2} \prod_{r=1}^{p} \Gamma(r / 2)} .
$$

We can now prove

Theorem 3. Let $f(S)$ be a function of the distinct elements of the $p$-rowed symmetric matrix $S$, such that $f(S)$ is a function only of the roots $\theta_{1}, \ldots, \theta_{p}$ of $S, \theta_{1} \geqq \theta_{2} \geqq \ldots \geqq \theta_{p}$ :

$$
f(S)=g\left(\theta_{1}, \ldots, \theta_{p}\right) \text {. }
$$

Let $\Sigma$ be the $\frac{1}{2} p(p+1)$-dimensional space. Then

$$
\int_{\Sigma} f(S) d m=\frac{\pi^{p(p+1) / 4}}{\prod_{,=1}^{p} \Gamma(r / 2)} \int_{\theta} g\left(\theta_{1}, \ldots, \theta_{p}\right) \prod_{i<j}\left(\theta_{i}-\theta_{j}\right) d \theta_{1} \ldots d \theta_{p},
$$

where $\Theta$ is the domain $\theta_{1}>\theta_{2}>\ldots>\theta_{p}$.

Proof. By the remark at the end of $\$ 2$ we have

$$
\int_{\Sigma} f(S) d m=\int_{E} f(S) d m .
$$


By Theorem 2 we may use the transformation (4), with the Jacobian (16). Hence

$\int_{\Sigma} f(S) d m=2^{2 p(p-1)} \int_{E^{*}}|I+X|^{-(p-1)} g\left(\theta_{1}, \ldots, \theta_{p}\right) \prod_{i<j}\left(\theta_{i}-\theta_{j}\right) d m$ where $E^{*}=M \times \Theta$. Hence

$\int_{\Sigma} f(S) d m=2^{\star} p(p-1) B_{p} \int_{\Theta} g\left(\theta_{1}, \ldots, \theta_{p}\right) \underset{i<}{\Pi}\left(\theta_{i}-\theta_{j}\right) d \theta_{1}, \ldots d \theta_{p}$.

Using (24) we get (25).

THEOREM 3 implies the following theorem on the probability distribution of roots, which is an important subject in statistics.

Theorem 4. Let the distinct elements of the p-rowed symmelric matrix $S$ be random variables whose joint distribution in $\Sigma$ has a probability density $f\left(S^{\prime}\right)$, such that $f(S)$ is a function only of the roots $\theta_{1} \geqq \ldots \geqq \theta_{p}$ of $S$ :

$$
f(S)=g\left(\theta_{1}, \ldots, \theta_{p}\right) .
$$

Then the joint distribution of roots in $\Theta$ has the probability density function

$$
\prod_{r=1}^{\frac{\pi^{p(p+1) / 4}}{p} \Gamma(r / 2)} g\left(\theta_{1}, \ldots, \theta_{p}\right) \prod_{i<j}\left(\theta_{i}-\theta_{j}\right) .
$$

Proof. If $A$ is any sub-set of $\Theta$, let $B$ be the sub-set of $\Sigma$ such. that $\left(\theta_{1}, \ldots, \theta_{p}\right) \in A$. Then

$$
\left.\operatorname{Pr}\left\{\theta_{1}, \ldots, \theta_{p}\right) \in A\right\}=\int_{\leq} \phi(S) f(S) d m,
$$

where $\phi(S)=1$ or 0 according as $S \epsilon B$ or $S \bar{\epsilon} B$. Now $\phi(S)=\psi\left(\theta_{1}, \ldots, \theta_{p}\right)$, where $\psi=1$ or 0 according as $\left(\theta_{1}, \ldots, \theta_{p}\right) \epsilon A$ or not. Hence $\phi(S) f(S)=\psi\left(\theta_{1}, \ldots, \theta_{p}\right) g\left(\theta_{1}, \ldots, \theta_{p}\right)$. Applying Theorem 3 . to (26) we get

$$
\begin{aligned}
& \operatorname{Pr}\left\{\left(\theta_{1}, \ldots, \theta_{p}\right) \in A\right\} \\
= & \prod_{\substack{p \\
\prod^{p(p+1) / 4}}}^{p} \int_{(r / 2)} \psi\left(\theta_{1}, \ldots, \theta_{p}\right) g\left(\theta_{1}, \ldots, \theta_{p}\right) \prod_{i<j}\left(\theta_{i}-\theta_{j}\right) d \theta_{1} \ldots d \theta_{p} \\
= & \frac{\pi^{p(p+1) / 4}}{p} \int_{r=1}^{p} g\left(\theta_{1}, \ldots, \theta_{p}\right) \prod_{i<j}\left(\theta_{i}-\theta_{j}\right) d \theta_{1} \ldots d \theta_{p},
\end{aligned}
$$

which proves the theorem.

University of North Carolina,

U.S.A. 